\title{
Existence of high energy solutions for Kirchhoff-type equations
}

\author{
Chunhan Liu ${ }^{1, \text { a }}$, Jianguo Wang ${ }^{2, b}$ \\ ${ }^{1}$ Department of Mathematics, Qilu Normal University, Jinan 250013, PR. China. \\ ${ }^{2}$ Department of Mathematics, Qilu Normal University, Jinan 250013, PR. China. \\ achh-liu1981@163.com, ${ }^{\mathrm{b}}$ jgw0801@163.com
}

Keywords: Nonlinear kirchhoff nonlocal equations, Fountain theorems, High energy solutions.

Abstract. In this paper, by applying the fountain theorems, we study the existence of infinitely many high energy solutions for the nonlinear kirchhoff nonlocal equations under the Ambrosetti-Rabinowitz type growth conditions or no Ambrosetti-Rabinowitz type growth conditions, infinitely many high energy solutions are obtained.

\section{Introduction and Main Results}

Recently, many authors studied Kirchhoff type problems, some important and interesting results can be found in [1-8]. In this paper, we study the following Kirchhoff-type problems

$$
\begin{cases}-\left(a+b \int_{\Omega}|\nabla u|^{2} d x\right) \Delta u+q(x) u=f(x, u), & \text { in } \Omega, \\ u=0, & \text { on } \partial \Omega,\end{cases}
$$

Where $\Omega$ is a smooth bounded domain in $R^{N}(N=1,2$ or 3$), a, b>0$, and $f: \Omega \times R \rightarrow R$ is continuous function.

Set $F(x, u)=\int_{0}^{u} f(x, s) d s$, Then a weak solution of problem (1) is a critical point of the following functional :

$$
\Phi(u)=\frac{a}{2} \int_{\Omega}|\nabla u|^{2} d x+\frac{b}{4}\left(\int_{\Omega}|\nabla u|^{2} d x\right)^{2}+\frac{1}{2} \int_{\Omega} q(x) u^{2} d x-\int_{\Omega} F(x, u) d x .
$$

In order to establish multiple solutions for problem (1), we make the following assumptions:

$\left(L_{1}\right) \quad \Omega \subset R^{N}$ is bound and open and, $q \in L^{\infty}(\Omega)$ and $q(x) \geq 0$ a.e. in $\Omega$.

$\left(L_{2}\right) f \in C(\bar{\Omega} \times R, R)$ and for some $4<p<2^{*}=\left\{\begin{array}{l}2 n / n-2 \quad n \geq 3 \\ +\infty \quad n=1,2,\end{array}\right.$ such that $|f(x, u)| \leq C(1+$ $\left.|u|^{p-1}\right)$

$\left(L_{3}\right) \lim _{|u| \rightarrow \infty}\left(f(x, u) u /|u|^{4}\right)=+\infty$.

$\left(L_{4}\right)$ There exists $\mu>4, R>0$, such that $|u| \geq R \Rightarrow 0<\mu F(x, u) \leq u f(x, u)$.

$\left(L_{4}^{\prime}\right)$ There is $c_{*} \geq 0, \theta \geq 1, H(x, t) \leq \theta H(x, s)+c_{*}$. for all $0<t<s, \forall x \in \Omega$, where $H(x, u)=$ uf $(x, u)-4 F(x, u)$

$$
\left(L_{5}\right) \quad f(x,-u)=-f(x, u) \text {. }
$$

Before stating our main results, we first introduce some preliminary nations. Let $E$ be a Banach space with the norm $\|\bullet\|$ and $E=\overline{\oplus_{j \in N} X_{j}}$ with $\operatorname{dim} X_{j}<\infty$ for any $j \in N$.Set $Y_{k}=\bigoplus_{j \in 0}^{k} X_{j} ; \quad Z_{k}=\overline{\oplus_{j=k}^{\infty} X_{j}}$ and $B_{k}=\left\{u \in Y_{k}:\|u\| \leq \rho_{k}\right\}, N_{k}=\left\{u \in Y_{k}:\|u\| \leq \gamma_{k}\right\}$ for $\rho_{k}>\gamma_{k}>0$.

- theorem1 ([1] Fountain theorem). Let $\varphi \in C^{1}(E, R)$ be a even functional. If for every $k \in N$, there exist $\rho_{k}>\gamma_{k}>0$. such that 
(A) $\quad a_{k}=\max _{u \in Y_{k},\|u\|=\rho_{k}} \varphi(u) \leq 0 ; \quad\left(A_{2}\right) \quad b_{k}=\inf _{u \in Z_{k},\|u\|=\gamma_{k}} \varphi(u) \rightarrow \infty, k \rightarrow \infty ; \quad\left(A_{3}\right) \quad \varphi$ satisfies the $(C)_{c}$ condition for every $c>0$, then $\varphi$ has an unbounded sequence of critical values.

Definition 1 Let $\Phi \in C^{1}(E, R)$,we say that $\Phi$ satisfies the cerami condition at the level $c \in R$, if any sequence $\left\{u_{n}\right\} \subset X$.along with

$\Phi\left(u_{n}\right) \rightarrow c$ and $\left(1+\left\|u_{n}\right\|\right) \Phi^{\prime}\left(u_{n}\right) \rightarrow 0$, as $n \rightarrow \infty$.

possesses a convergent subsequence; $\Phi$ satisfies the $(C)$ condition if $\Phi$ satisfies $(C)_{c}$ for all $c \in R$.

In this paper, we consider $E=H_{0}^{1}(\Omega)$ endowed with the norm $\|u\|_{E}=\left(\int_{\Omega}|\nabla u|^{2} d x+\int_{\Omega} q(x) u^{2} d x\right)^{\frac{1}{2}}$.

$L^{P}(\Omega)$ denotes the usual Lebesgue space with the norm $|u|_{p}=\left(\int_{\Omega}|u|^{p} d x\right)^{\frac{1}{p}}$. Since $\Omega$ is a bounded domain, it is well known that $E \hookrightarrow L^{p}(\Omega)$ continuously for $p \in\left[1,2^{*}\right]$, and compactly for $p \in\left[1,2^{*}\right]$. Hence,for $p \in\left[1,2^{*}\right]$, there exists $\gamma_{p}$ such that $|u|_{p} \leq \gamma_{p}\|u\|_{E}, \forall u \in E$.

The main results of this paper are the following:

Theorem 2 Suppose that $\left(L_{1}\right)\left(L_{2}\right)\left(L_{4}\right)\left(L_{5}\right)$ hold, Then problem (1) has a sequence of solutions $\left\{u_{n}\right\}$ such that $\Phi\left(u_{n}\right) \rightarrow \infty$, as $n \rightarrow \infty$.

Theorem 3 Suppose that $\left(L_{1}\right)-\left(L_{3}\right)\left(L^{\prime}{ }_{4}\right)\left(L_{5}\right)$ hold, Then problem (1) has a sequence of solutions $\left\{u_{n}\right\}$ such that $\Phi\left(u_{n}\right) \rightarrow \infty$, as $n \rightarrow \infty$.

\section{Proofs of Theorems}

Proof of Theorem 2 (i) Let $\left\{u_{n}\right\} \subset E$ be a $(C)_{c}$ sequence of $\Phi(u)$. Then for $n$ large enough, we have

$$
\begin{aligned}
& C\left(1+\left\|u_{n}\right\|\right) \geq \Phi\left(u_{n}\right)-\frac{1}{\mu} \Phi^{\prime}\left(u_{n}\right) u_{n} \quad=a\left(\frac{1}{2}-\frac{1}{\mu}\right) \int_{\Omega}\left|\nabla u_{n}\right|^{2} d x+b\left(\frac{1}{4}-\frac{1}{\mu}\right)\left(\int_{\Omega}\left|\nabla u_{n}\right|^{2} d x\right)^{2} \\
+ & \left(\frac{1}{2}-\frac{1}{\mu}\right) \int_{\Omega} q(x) u_{n}^{2} d x-\int_{\Omega} F\left(x, u_{n}\right) d x+\frac{1}{\mu} \int_{\Omega} f\left(x, u_{n}\right) u_{n} d x . \\
& \geq \min \{a, 1\}\left(\frac{1}{2}-\frac{1}{\mu}\right)\|u\|_{E}^{2}+\frac{1}{\mu}\left[\int_{\Omega}-\mu F\left(x, u_{n}\right) d x+\int_{\Omega} f\left(x, u_{n}\right) u_{n} d x\right] .
\end{aligned}
$$

we obtain from $\left(L_{2}\right)$ that

$\int_{\Omega}\left[-\mu F\left(x, u_{n}\right)+f\left(x, u_{n}\right) u_{n}\right] d x \geq \int_{\left|u_{n}\right| \leq R}\left[-\mu F\left(x, u_{n}\right)+f\left(x, u_{n}\right) u_{n} d x \geq-C_{1}\right.$.

we have $C\left(1+\left\|u_{n}\right\|\right) \geq \min \{a, 1\}\left(\frac{1}{2}-\frac{1}{\mu}\right)\left\|u_{n}\right\|_{E}^{2}-C_{1}$.

which is course implies that $\left\{u_{n}\right\}$ is bounded. We have $u_{n} \rightarrow u$ in $H_{0}^{1}(\Omega), u_{n} \rightarrow u$ in $L^{p}(\Omega)$. Then Theorem A.2 ${ }^{[1]}$ (willem 1996) implies that $f\left(x, u_{n}\right) \rightarrow f(x, u)$ in $L^{q}(\Omega), q=p / p-1$.we next prove that $\left\{u_{n}\right\}$ has a convergent subsequence obverse that

$$
\begin{gathered}
\left\langle\Phi^{\prime}\left(u_{n}\right)-\Phi^{\prime}(u), u_{n}-u\right\rangle=a \int_{\Omega}\left|\nabla\left(u_{n}-u\right)\right|^{2} d x+b \int_{\Omega}\left|\nabla u_{n}\right|^{2} d x \int_{\Omega} \nabla u_{n} \nabla\left(u_{n}-u\right) d x+b \int_{\Omega}|\nabla u|^{2} d x \int_{\Omega} \nabla u \nabla\left(u_{n}-u\right) d x \\
+b \int_{\Omega}|\nabla u|^{2} d x \int_{\Omega} \nabla u \nabla\left(u-u_{n}\right) d x+\int_{\Omega} q(x)\left|u_{n}-u\right|^{2} d x-\int_{\Omega}\left(f\left(x, u_{n}\right)-f(x, u)\right)\left(u_{n}-u\right) d x \\
\quad \geq \min \{a, 1\}\left\|u_{n}-u\right\|_{E}^{2}+b \int_{\Omega}\left(\left|\nabla u_{n}\right|^{2}-|\nabla u|^{2}\right) d x \int_{\Omega} \nabla u \nabla\left(u_{n}-u\right) d x \\
\quad-\int_{\Omega}\left(f\left(x, u_{n}\right)-f(x, u)\right)\left(u_{n}-u\right) d x
\end{gathered}
$$

using the boundedness of $\left\{u_{n}\right\}$ and $u_{n} \rightarrow u$ in $H_{0}^{1}(\Omega)$, one has 


$$
b \int_{\Omega}\left(\left|\nabla u_{n}\right|^{2}-|\nabla u|^{2}\right) d x \int_{\Omega} \nabla u \nabla\left(u_{n}-u\right) d x \rightarrow 0, \text { as } n \rightarrow \infty .
$$

It follows from the Höld inequality that $\int_{\Omega}\left(f\left(x, u_{n}\right)-f(x, u)\right)\left(u_{n}-u\right) d x \leq\left|f\left(x, u_{n}\right)-f(x, u)\right|_{q}\left|u_{n}-u\right|_{p}$. as $n \rightarrow \infty$.

Thus we deduce that $\left\|u_{n}-u\right\|_{E} \rightarrow 0$ as $n \rightarrow \infty$. Hence $\Phi$ satisfies the $(C)_{c}$ condition for every $c>0$.

(ii) By $\left(L_{4}\right)$,we have $F(x, u) \geq C\left(|u|^{\mu}-1\right)$.

$$
\begin{aligned}
\Phi(u)=\frac{a}{2} \int_{\Omega} & |\nabla u|^{2} d x+\frac{b}{4}\left(\int_{\Omega}|\nabla u|^{2} d x\right)^{2}+\frac{1}{2} \int_{\Omega} q(x) u^{2} d x-\int_{\Omega} F(x, u) d x \\
& \leq \frac{a}{2} \int_{\Omega}|\nabla u|^{2} d x+\frac{b}{4}\left(\int_{\Omega}|\nabla u|^{2} d x\right)^{2}+\frac{1}{2} \int_{\Omega} q(x) u^{2} d x-C \int_{\Omega}|u|^{\mu} d x+\int_{\Omega} C d x \\
& \leq \max \{a, 1\}\|u\|_{E}^{2}+\frac{b}{4}\|u\|_{E}^{2}-C \int_{\Omega}|u|^{\mu} d x+C \Omega .
\end{aligned}
$$

Since on the finite-dimensional space $Y_{k}$ all norms are equivalent, there for $\left(A_{1}\right)$ is satisfied for every $\rho_{k}>0$ large enough.

(iii) We next verify condition $\left(A_{3}\right)$, To this end,from $\left(L_{2}\right)$, we have $|F(x, u)| \leq C_{1}\left(1+|u|^{p}\right)$.

Define $\beta_{k}=\max _{u \in Z_{k},\|u\|_{E}=1}|u|^{p}$. so that on $Z_{k}$, we have

$$
\begin{aligned}
& \Phi(u)=\frac{a}{2} \int_{\Omega}|\nabla u|^{2} d x+\frac{b}{4}\left(\int_{\Omega}|\nabla u|^{2} d x\right)^{2}+\frac{1}{2} \int_{\Omega} q(x) u^{2} d x-\int_{\Omega} F(x, u) d x \\
& \geq \frac{1}{2} \min \{a, 1\}\|u\|_{E}^{2}-C_{1}|u|_{p}^{p}-C_{2} \geq \frac{1}{2} \min \{a, 1\}\|u\|_{E}^{2}-C_{1} \beta_{k}^{p}\|u\|_{E}^{p}-C_{2} .
\end{aligned}
$$

Take $\gamma_{k}=\left(C_{1} \rho \beta_{k}^{p} / \sqrt{\min \{a, 1\} / 2}\right)^{\frac{1}{2-p}}$, since $\beta_{k} \rightarrow 0, k \rightarrow \infty$. we obtain for $u \in Z_{k}$, with $\|u\|_{E}=r_{k}$.

$\Phi(u) \geq \frac{1}{2} \min \{a, 1\}\left(C_{1} \rho \beta_{k}^{p} / \sqrt{\min \{a, 1\} / 2}\right)^{\frac{2}{2-p}}-C_{2} \rightarrow \infty$, as $k \rightarrow \infty$.

So condition $\left(A_{2}\right)$ is proved. Now all conditions of Theorem 1 hold. Therefore, problem(1) has a sequence of solutions $\left\{u_{n}\right\}, \Phi\left(u_{n}\right) \rightarrow \infty$, as $n \rightarrow \infty$.

Proof of Theorem 3 (i) We verify $\Phi$ satisfies $(C)_{c}$ for all $c \in R$, such that

$$
\Phi\left(u_{n}\right) \rightarrow c \text { and }\left(1+\left\|u_{n}\right\|\right) \Phi^{\prime}\left(u_{n}\right) \rightarrow 0 \text {, as } n \rightarrow \infty .
$$

we first show that $\left\{u_{n}\right\}$ is bound, which is our main propose. Indeed, suppose that $\left\|u_{n}\right\|_{E} \rightarrow \infty$, setting $v_{n}=u_{n} /\left\|u_{n}\right\|_{E}$, then $\left\|v_{n}\right\|_{E}=1$, so we can suppose that

$v_{n} \rightarrow v$ in $H_{0}^{1}(\Omega), v_{n} \rightarrow v$ in $L^{p}(\Omega), v_{n} \rightarrow v$ a.e. $x \in \Omega$.

Next we consider the two possible cases:(1) $v \neq 0$, (2) $v=0$.

In $\quad(1)$, wase he $o(1)=\left\langle\Phi^{\prime}\left(u_{n}\right), u_{n}\right\rangle$,

$\int_{\Omega}\left(f\left(x, u_{n}\right) u_{n} /\left\|u_{n}\right\|_{E}^{4}\right) d x \leq \max \{a, 1\} /\left\|u_{n}\right\|_{E}^{4}+b-o(1) \leq C<+\infty$.

On the other hand,on the set $\sum=\{x \in \Omega \mid v(x) \neq 0\} . f\left(x, u_{n}\right) u_{n} /\left\|u_{n}\right\|_{E}^{4}=\left(f\left(x, u_{n}\right) u_{n} / u_{n}{ }^{4}\right) v_{n}{ }^{4} \rightarrow+\infty$, as $n \rightarrow \infty$.

by $\left(L_{2}\right)$ for any $M>0$, there exist $C_{1}>0$, such that $f\left(x, u_{n}\right) \geq M|u|^{3}-C_{1}$, for a.e. $x \in \Omega$, and $n \in N$.

$\lim _{n \rightarrow \infty} \int_{\Omega} f\left(x, u_{n}\right) u_{n} /\left\|u_{n}\right\|_{E}^{4} d x=\lim _{n \rightarrow \infty} \int_{\Omega}\left(f\left(x, u_{n}\right) u_{n} / u_{n}{ }^{4}\right) v_{n}{ }^{4} d x \geq \lim _{n \rightarrow \infty}\left(M \int_{\Omega} v_{n}{ }^{4} d x-\left(C_{1} /\left\|u_{n}\right\|_{E}^{3}\right) \int_{\Omega} v_{n} d x=M \int_{\Omega} v^{4} d x\right.$

Since $m \sum>0$, we infer that $\lim _{n \rightarrow \infty} \int_{\Omega}\left(f\left(x, u_{n}\right) u_{n} /\left\|u_{n}\right\|_{E}^{4} d x\right) \rightarrow \infty$, as $n \rightarrow \infty$. which contradicts to (3). 
In case (2),we can define $\Phi\left(t_{n} u_{n}\right)=\max _{t \in[0,1]} \Phi\left(t u_{n}\right)$, For any give $R>0$, define $\bar{v}_{n}=R\left(u_{n} /\left\|u_{n}\right\|_{E}\right)=R v_{n}$, from $\left(L_{2}\right)$, we have

$$
\begin{array}{ccc}
\int_{\Omega} F\left(x, \bar{v}_{n}\right) \leq C \int_{\Omega}\left|\bar{v}_{n}\right| d x+C \int_{\Omega}\left|\bar{V}_{n}\right|^{p} d x \rightarrow 0 . & \text { as } & n \rightarrow \infty . \\
\Phi\left(t_{n} u_{n}\right) \geq \frac{a}{2} \int_{\Omega}\left|\nabla \bar{v}_{n}\right|^{2} d x+\frac{b}{4}\left(\int_{\Omega}\left|\nabla \bar{v}_{n}\right|^{2} d x\right)^{2}+\frac{1}{2} \int_{\Omega} q(x) \bar{v}_{n}^{2} d x-\int_{\Omega} F\left(x, \bar{v}_{n}\right) d x & \\
\geq\left.\frac{1}{2} \min \{a, 1\}||_{v_{n}}\right|_{E} ^{2}+\frac{b}{4}\left(\int_{\Omega}\left|\nabla \bar{v}_{n}\right|^{2} d x\right)^{2}-\int_{\Omega} F\left(x, \bar{v}_{n}\right) d x \geq C R .
\end{array}
$$

which means that $\lim _{n \rightarrow \infty} \Phi\left(t_{n} u_{n}\right)=\infty$. By the definition of $t_{n}$, we see that $o(1)=\left\langle\Phi^{\prime}\left(u_{n}\right), u_{n}\right\rangle$ consequently, By $\left(L^{\prime}{ }_{4}\right)$,we have

$$
\begin{aligned}
\infty & \leftarrow 4 \Phi\left(t_{n} u_{n}\right)-\left\langle\Phi^{\prime}\left(t_{n} u_{n}\right), t_{n} u_{n}\right\rangle=a \int_{\Omega}\left|\nabla\left(t_{n} u_{n}\right)\right|^{2} d x+\int_{\Omega} q(x)\left(t_{n} u_{n}\right)^{2} d x+\int_{\Omega}\left[f\left(x, t_{n} u_{n}\right) t_{n} u_{n}-4 F\left(x, t_{n} u_{n}\right)\right] d x \\
\leq & a \int_{\Omega}\left|\nabla u_{n}\right|^{2} d x+\int_{\Omega} q(x) u_{n}^{2} d x+\theta \int_{\Omega}\left[f\left(x, u_{n}\right) u_{n}-4 F\left(x, u_{n}\right)\right] d x+C_{*} \\
& \leq \theta\left[4 \Phi\left(u_{n}\right)-\left\langle\Phi^{\prime}\left(u_{n}\right), u_{n}\right\rangle\right]+C_{*}<C .
\end{aligned}
$$

as $n \rightarrow \infty$, which is a contradiction. This proves that $\left\{u_{n}\right\}$ is bounded.

$u_{n} \rightarrow u$ in $H_{0}^{1}(\Omega), u_{n} \rightarrow u$ in $L^{p}(\Omega), u_{n} \rightarrow u$ a.e. $x \in \Omega$.

By Theorem 1 (i) We have $\left\|u_{n}-u\right\|_{E} \rightarrow 0$, as $n \rightarrow \infty$. Thus $u_{n} \rightarrow u$ strongly in $H_{0}^{1}(\Omega)$, which means that $\Phi$ satisfies $(C)_{c}$.

(ii) By $\left(L_{3}\right)$,we have $F(x, u) \geq M|u|^{4}-C_{1}|u|$,

$$
\begin{gathered}
\Phi(u) \leq \frac{a}{2} \int_{\Omega}|\nabla u|^{2} d x+\frac{b}{4}\left(\int_{\Omega}|\nabla u|^{2} d x\right)^{2}+\frac{1}{2} \int_{\Omega} q(x) u^{2} d x-M \int_{\Omega}|u|^{4} d x+C_{1} \int_{\Omega}|u| d x \\
\leq \max \{a, 1\}\|u\|_{E}^{2}+(b / 4)\|u\|_{E}^{2}-M C\|u\|_{E}^{4}+C_{1} \int_{\Omega}|u| d x .
\end{gathered}
$$

Since on the finite-dimensional space $Y_{k}$ all norms are equivalent,we take $M$ large enough, such that $b / 4-M C<0$, there for $\left(A_{1}\right)$ is satisfied for every $\rho_{k}>0$ large enough.

By Theorem 1 (iii), condition $\left(A_{2}\right)$ is proved. Now all conditions of Theorem 1 hold, therefore, problem (1) has a sequence of solutions $\left\{u_{n}\right\}$, such that $\Phi\left(u_{n}\right) \rightarrow \infty$, as $n \rightarrow \infty$.

\section{References}

[1] M. Willem, Minimax Theorems, Birkhäuser, Boston, 1996.

[2] X. He, W. Zou, Infinitely many positive solutions for Kirchhoff-type problems, Nonlinear Anal,70, 1407-1414 (2009).

[3]X. He, W. Zou, Multiplicity of solutions for a class of Kirchhoff type problems, Sin.Acta.Math. Appl, 26 (2010), 387-394.

[4]A. Mao, Z. Zhang. Sign-changing and multiple solutions of Kirchhoff type problems without the P.S. condition, Nonlinear Anal, 70(3), 1275-1287 (2009).

[5] K. Perera, Z. Zhang, Nontrivial solutions of Kirchhoff-type problems via the Yang index, J. Differ. Equ, 221(1), 246-255 (2006).

[6] Z. Zhang, K. Perera. Sign changing solutions of Kirchhoff type problems via invariant sets of descent flow, J. Math. Anal.Appl. 317 (2) , 456-463 (2006).

[7] Nguyen Lam, G. Lu, Elliptic equations and systems with subcritical and critical exponential growth with- 
out the ambrosetti-rabinowitz condition, J. Geom. Anal. (2012).

[8] Cheng, B, Wu, X, Existence results of positive solutions of Kirchhoff type problems, Nonlinear Anal. 71, $4883-4892$ (2009).

[9] Alves, C.O, Corrëa, F.J.S.A, Ma, T.F, Positive solutions for a quasilinear elliptic equation of Kirchhoff type, Comput. Math.Appl. 49,85-93 (2005).

[10] W .Zou, M. Schechter, Critical point theory and its applications,Springer, New York. (2006). 